\title{
Article
}

\section{Bacterial Footprints in Elastic Pillared Microstructures}

Susarrey-Arce, Arturo, Hernández-Sánchez, José Federico, Marcello, Marco, Diaz-Fernandez, Yuri, Oknianska, Alina, Sorzabal-Bellido, Ioritz, Tiggelaar, Roald, Lohse, Detlef, Gardeniers, Han, Snoeijer, Jacco, Marin, Alvaro and Raval, Rasmita

Available at https://clok.uclan.ac.uk/33618/

Susarrey-Arce, Arturo, Hernández-Sánchez, José Federico, Marcello, Marco, Diaz-Fernandez, Yuri, Oknianska, Alina, Sorzabal-Bellido, loritz, Tiggelaar, Roald, Lohse, Detlef, Gardeniers, Han et al (2018) Bacterial Footprints in Elastic Pillared Microstructures. ACS Applied Bio Materials, 1 (5). pp. 12941300. ISSN 2576-6422

It is advisable to refer to the publisher's version if you intend to cite from the work. http://dx.doi.org/10.1021/acsabm.8b00176

For more information about UCLan's research in this area go to http://www.uclan.ac.uk/researchgroups/ and search for < name of research Group>.

For information about Research generally at UCLan please go to

http://www.uclan.ac.uk/research/

All outputs in CLoK are protected by Intellectual Property Rights law, including Copyright law. Copyright, IPR and Moral Rights for the works on this site are retained by the individual authors and/or other copyright owners. Terms and conditions for use of this material are defined in the policies page. 


\title{
Bacterial Footprints in Elastic Pillared Microstructures
}

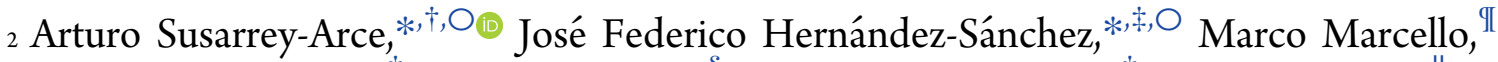 \\ ${ }_{3}$ Yuri Diaz-Fernandez, ${ }^{\dagger}$ Alina Oknianska, ${ }^{\S}$ Ioritz Sorzabal-Bellido, ${ }^{\dagger}$ Roald Tiggelaar, ${ }^{\|}$Detlef Lohse, ${ }^{\perp}$ (0) \\ ${ }_{4}$ Han Gardeniers, ${ }^{\#}$ J Jacco Snoeijer, ${ }^{*}{ }^{\perp}$ Alvaro Marin, ${ }^{\perp}$ and Rasmita Raval ${ }^{*}{ }^{\dagger}$ \\ $5{ }^{\dagger}$ Open Innovation Hub for Antimicrobial Surfaces at the Surface Science Research Centre and Department of Chemistry, University \\ 6 of Liverpool, Oxford Street, Liverpool L69 3BX, United Kingdom \\ 7 Division of Physical Sciences and Engineering and Clean Combustion Research Center, King Abdullah University of Science and \\ 8 Technology, Thuwal 23955-6900, Saudi Arabia \\ 9 II Institute of Integrative Biology, University of Liverpool, Biosciences Building, Liverpool L69 7ZB, United Kingdom \\ $10{ }^{\S}$ School of Health Sciences, Liverpool Hope University, Hope Park, Liverpool L16 9JD, United Kingdom \\ ${ }_{11}$ "NanoLab Cleanroom, MESA+ Institute for Nanotechnology, University of Twente, P.O. Box 217, Enschede 7500AE, The \\ 12 Netherlands \\ ${ }_{13}{ }^{\perp}$ Physics of Fluids Group, MESA+ Institute for Nanotechnology, J.M. Burgers Centre for Fluid Dynamics, University of Twente, \\ 14 P.O. Box 217, Enschede 7500AE, The Netherlands \\ 15 " Mesoscale Chemical Systems, MESA+ Institute for Nanotechnology, University of Twente, P.O. Box 217, Enschede 7500AE, The \\ 16 Netherlands
}

\section{S Supporting Information}

18 ABSTRACT: Soft substrates decorated with micropillar arrays are known to

19 be sensitive to deflection due to capillary action. In this work, we demonstrate

20 that micropillared epoxy surfaces are sensitive to single drops of bacterial

21 suspensions. The micropillars can show significant deformations upon 22 evaporation, just as capillary action does in soft substrates. The phenomenon

23 has been studied with five bacterial strains: S. epidermidis, L. sakei, P. 24 aeruginosa, E. coli, and B. subtilis. The results reveal that only droplets 25 containing motile microbes with flagella stimulate micropillar bending, which 26 leads to significant distortions and pillar aggregations forming dimers, trimers, 27 and higher order clusters. Such deformation is manifested in characteristic 28 patterns that are left on the microarrayed surface following evaporation and 29 can be easily identified even by the naked eye. Our findings could lay the 30 ground for the design and fabrication of mechanically responsive substrates, 31 sensitive to specific types of microorganisms.

32 KEYWORDS: bacteria, bending, elastic micropillars, capillarity, responsive substrates

\section{Stimulated bending by bacterial species}

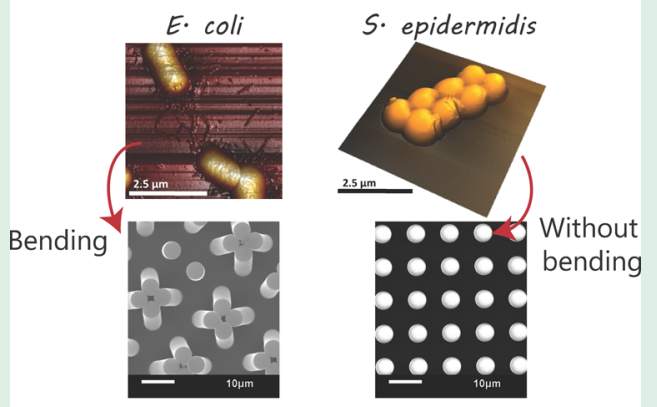

Substrates decorated with micropillars
34 The fabrication of materials that are sensitive to physical, 35 chemical, or biological stimuli has opened opportunities for the 36 development of a wide variety of technological applications 37 such as switchable adhesion, mechanosensing, and stimuli38 responsive materials. ${ }^{1-6}$ In particular, the design of biomimetic 39 structures, ${ }^{3,7}$ inspired by natural systems, has been a powerful 40 tool in the implementation of smart, artificial systems. ${ }^{8,9}$ In this 41 respect, the use of topographic surfaces is particularly 42 interesting, with natural systems utilizing physical structures, 43 from the nano- to the macroscale, to deliver functions such as 44 superhydrophobicity, adhesion, and antibiofouling as demon45 strated by the lotus leaf, shark skin, and gecko feet. ${ }^{4,7,9-13}$

46 There has been particular interest in developing mechan47 ically responsive systems. ${ }^{8,14}$ An excellent example is the 48 mechanical response of micropillar arrays upon drying of water (or water-based solutions). ${ }^{15-26}$ When water droplets 49 evaporate on relatively soft elastic microstructured surfaces, 50 capillary action can generate a significant force that is able to 51 bend the soft micropillars. Depending on the geometry of the 52 arrays, the capillary and elastic forces can form different pillar 53 assemblies. $^{15,16}$ The complexity of the assemblies varies with 54 the pillar height and the interpillar distance. For example, large 55 periodic chiral aggregates can be formed when the micropillars 56 are higher and closer to each other. Each cluster of aggregates 57 has a different potential to store elastic energy, embody 58 information, enhance adhesion, or capture particles. ${ }^{17,18} \quad 59$

Received: $\quad$ May 31, 2018

Accepted: October 15, 2018

Published: October 15, 2018 
60 The demonstration of mechanically responsive topographic 61 surfaces to bacterial stimuli during evaporation of small 62 droplets is of great interest and has not been demonstrated 63 before. Furthermore, the deflections seen in our systems are 64 significant, leading to pillar aggregations into dimers, trimers, 65 and higher order clusters. Recently, the formation of biofilm 66 strings and networks between topographic pillars has been 67 demonstrated in liquid media; ${ }^{27}$ however, the mechanical 68 response of the pillars to bacterial presence upon evaporation 69 is not observed. Chew and coauthors have shown small 70 deflections of macropillared surfaces in response to the 71 differential pressure exerted by biofilm growth within a growth 72 chamber over a $24 \mathrm{~h}$ period, ${ }^{28}$ while Biais $^{29}$ and $\mathrm{Ng}^{30}$ et al. 73 have investigated the interaction of bacterial pili with pillared 74 structures.

75 Here, we demonstrate how epoxy-made soft surfaces 76 containing micropillar arrays interact with suspensions of 77 different bacterial species. Our results suggest that the presence 78 of motile bacteria with flagella drastically increases the 79 mechanical response of the pillars, actively bending soft 80 topographical substrates in the area contained within the 81 contact line. In contrast, solutions containing nonmotile 82 bacteria do not generate such responses. We attribute this to 83 the ability of motile bacteria to interact with each other and 84 with their topographical environment. Importantly, the 85 response of the microarray is sensitive to the type and 86 concentration of bacteria in the solution. These promising 87 results could lay the foundation for the development of devices 88 that are selectively responsive to specific microorganisms, 89 paving the way to construct smart, fast, and cost-effective 90 diagnostic tools.

\section{RESULTS AND DISCUSSION}

92 One of the key parameters in the mechanical response of soft 93 micropillar arrays is the aspect ratio of a single pillar. We 94 investigated the effect of the pillar aspect ratio by fabricating 95 regular patterns of cylindrical pillars with a constant diameter $96(5 \mu \mathrm{m})$ and interspacing $(5 \mu \mathrm{m})$ and with variable height 97 (from 5 to $45 \mu \mathrm{m}$ ). The patterns were created on epoxy resin 98 using a method described before ${ }^{31-35}$ based on casting 99 uncured epoxy on a negative polydimethilsiloxane (PDMS) 100 mold, followed by curing and mechanically removing of the 101 mold. The micropatterns were transferred efficiently, with a 102 high degree of fidelity, as shown by scanning electron 103 microscopy (SEM) imaging (Figure 1 and Figure S1).

104 These microstructured substrates can be susceptible to 105 elastocapillary forces in the presence of pure liquids. Therefore, 106 we evaluated the effect of pure water over a surface decorated 107 with micropillars with lengths varying from 5 to $45 \mu \mathrm{m}$ (Figure 108 1) during the evaporation of water droplets (Figure 1). In 109 these experiments, the liquid filled up the space between the 110 pillars, resulting in an almost square-shaped droplet contour. 111 Once the droplet spreads on the substrate, the liquid contact 112 line is blocked by the pillared structure and remains 113 immobilized (pinned) for the rest of the drying process. ${ }^{31}$ 114 Figure $1 \mathrm{~b}$ shows that after complete evaporation, there is 115 almost no trace of the droplet, except at the droplet contour, 116 where lines of pillars were bent by capillary action at the 117 contact line shown in Video $S 1 .^{18-23,31}$

118 In the systems studied, the pillar lattice was kept constant 119 (i.e., $l=d=5 \mu \mathrm{m}$ ), but different pillar heights $(h)$ ranging from $120 h=5$ to $45 \mu \mathrm{m}$ were fabricated. Thus, a range of 121 micropatterned surfaces were generated with different aspect
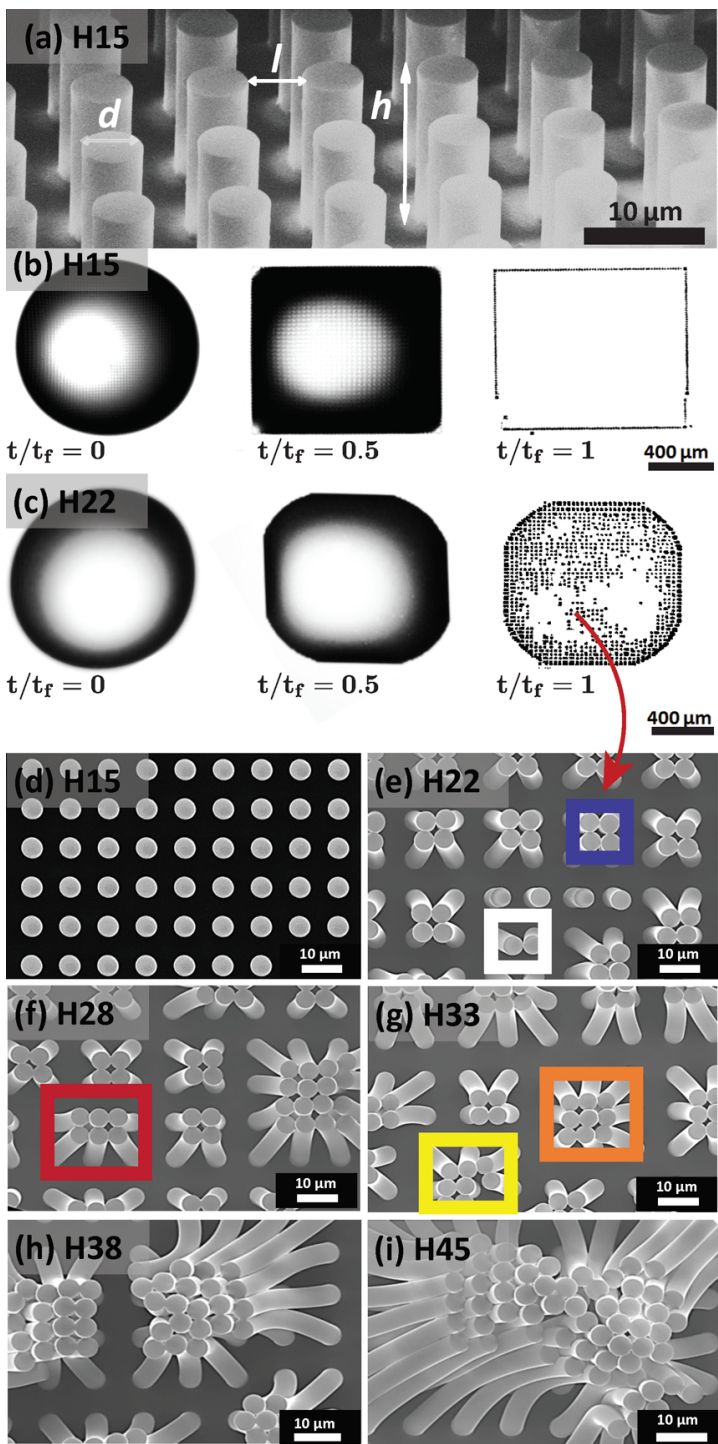

Figure 1. (a) Representative SEM image of pillared structure (H15), showing the topographic descriptors for the array. The pillars have a cylindrical shape and a height $(h)$ of $15 \mu \mathrm{m}$ and a diameter $(d)$ of 5 $\mu \mathrm{m}$ forming a square lattice with an interpillar distance $(l)=5 \mu \mathrm{m}$. (b) Pure water droplet evaporating on the H15 substrate with micropillars leaving a distinct square-shaped contact line with no perturbation of pillars within this contour. (c) Pure water droplet evaporating on the $\mathrm{H} 22$ substrate with micropillars leaving a distinct shaped contact line pattern with significant modification of the micropillars within the contact line boundary. Time needed is represented in a dimensionless form as the ratio between the elapsed time $(t)$ and the final evaporation time $\left(t_{\mathrm{f}}\right) .(\mathrm{d}-\mathrm{i})$ Pillared structures with constant $(d=5 \mu \mathrm{m})$ and different pillar heights $(h)$ of (d) $15 \mu \mathrm{m}$ (H15), (e) $22 \mu \mathrm{m}$ (H22), (f) $28 \mu \mathrm{m}$ (H28), (g) $33 \mu \mathrm{m}$ (H33), (h) 38 $\mu \mathrm{m}$ (H38), and (i) $45 \mu \mathrm{m}$ (H45). SEM images are presented for the different heights after evaporation of pure water droplets, probing the sensitivity of the structures to pure elastocapillary bending.

ratios (i.e., $h / d=3$ to $h / d=9$ ). For large aspect ratio 122 structures, we observed significant perturbation of the 123 micropillars in the area within the contact line boundary. 124 Imaging at low magnifications, or even examination by the 125 naked eye, revealed that the inner part of the pattern was 126 opaque, suggesting that the whole array of pillars inside the 127 dried droplet perimeter was modified (Figure 1c). Higher 128 magnification SEM imaging showed that this optical contrast 129 
130 effect was caused by local bending of the micropillars (Figure $1311 \mathrm{~d}-\mathrm{i}$ ), with the pillars bent toward each other forming clusters 132 and adopting complex geometries, e.g., dimer (white box), 133 tetramer (blue box), hexamer (red box), octamer (yellow box), 134 and nonamer (orange box). Similar effects have been reported 135 before for larger pillar aspect ratios ${ }^{18,24,25}$ and were attributed 136 to the elastocapillary coalescence of the flexible structures. ${ }^{15,18}$ 137 In our experiments, as the aspect ratio decreased, the clusters 138 contained lower numbers of aggregated pillars until a critical 139 aspect ratio $h / d=3$, for which no clusters were observed in the 140 inner part of the droplet (Figure 1d).

141 The deformation of the pillars, upon water evaporation, is 142 induced by the surface tension $(\gamma)$ of the water/air meniscus 143 connecting the pillars, and the corresponding force scales as $F_{c}$ $144 \sim \gamma r$, where $r=d / 2$ is the pillar radius. ${ }^{21,36}$ The natural 145 elasticity of the pillars resists deformation with an elastic force $146 F_{E} \sim E l r^{4} / h^{3}$, where $E$ is the Young modulus and $l$ the 147 interpillar distance. ${ }^{18}$ This expression is analogous to the usual 148 beam theory for slender objects, showing that the resistance to 149 bending decreases strongly when the pillars height increases. If 150 we define the pillar bending sensitivity as the ratio of capillary 151 and elastic forces, $F_{\mathrm{c}} / F_{\mathrm{E}}=\gamma / \mathrm{El}(h / r)^{3}$, we can conclude that it 152 is directly proportional to the cubic power of the pillar aspect 153 ratio $h / r$; i.e., slender pillars are more prone to be bent by 154 surface tension, while wide pillars tend to be more stable.

155 Under our experimental conditions, no pillar coalescence is 156 observed in the area within the contact line boundary from 157 pure water when the aspect ratio is below $h / d=3,{ }^{31}$ suggesting 158 that this is the critical aspect ratio threshold for which capillary 159 action equals restoration mechanical stress on the micropillars. 160 It is important to note that in this analysis, we are not 161 considering the effect of the contact line. This effect is 162 expected to have an enhanced deforming effect, but an 163 accurate evaluation of this factor is beyond existing 164 phenomenological modeling capabilities and will be the subject 165 of future studies. Consequently, all of the results described 166 below applies exclusively to the inner part of the dried pattern 167 left by the droplet, ignoring possible contact line effects.

168 Bacterial-Triggered Coalescence of Pillars. From the 169 elastocapillary assay discussed in the previous section, we 170 identified the critical region within the topographic parameter 171 space where the micropillared structure is able to resist 172 capillary deformation in the presence of pure water droplets. 173 Such a surface opens up the possibility to sense the presence of 174 a second entity introduced into water (i.e., bacterial cells), 175 which could induce a response in its own right. This critical 176 structure corresponds to an aspect ratio $h / d \approx 3$ and pillar 177 height $h=15 \mu \mathrm{m}$ (H15, Figure 1d), as discussed in the 178 previous section.

179 We, therefore, investigated the drying process of droplets 180 containing different bacteria species over the H15 pillared 181 structures. Similar to the case of pure water droplets, a pinned 182 square drop shape is found. However, the patterns observed 183 within the contact line formed after complete evaporation of 184 the droplets were surprisingly different for some bacteria as 185 clearly observed in Video S2.

186 Five different bacterial species, with a wide range of 187 morphological and biological characteristics were investigated: 188 S. epidermidis, L. sakei, P. aeruginosa, E. coli, and B. subtilis. The 189 patterns formed after evaporation of droplets containing 190 different bacteria on H15 pillar substrates (Figure 2) can be 191 classified in two main groups: one group displaying significant 192 bending of the pillars within the pattern (P. aeruginosa, E. coli,

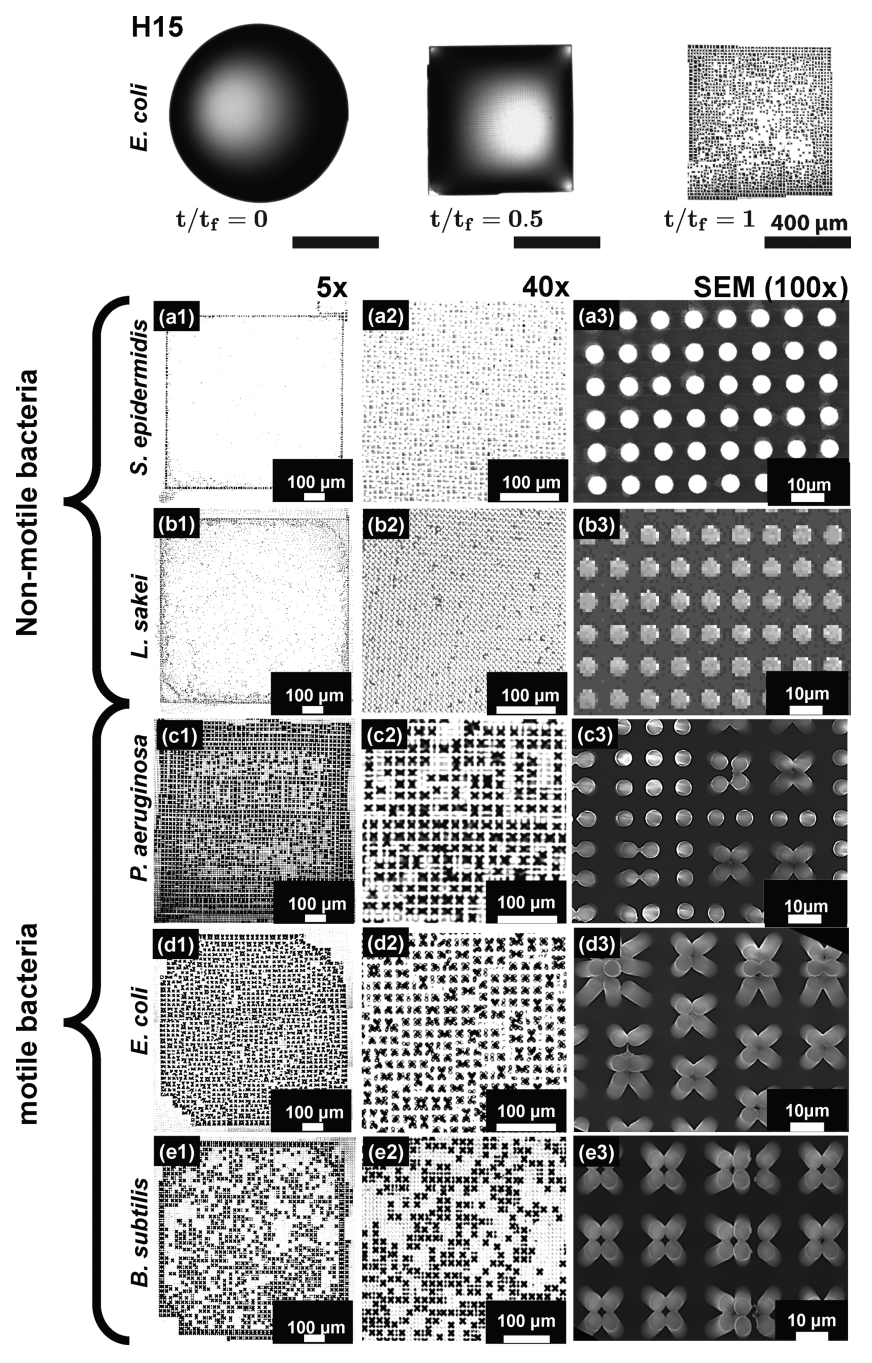

Figure 2. Typical patterns left over H15 substrates after the evaporation of different bacterial species: (a1-a3) S. epidermidis, (b1-b3) L. sakei, (c1-c3) P. aeruginosa, $(\mathrm{d} 1-\mathrm{d} 3)$, E. coli, $(\mathrm{e} 1-\mathrm{e} 3)$ B. subtilis. Here, the concentration of the different bacterial species is $10^{7}$ $\mathrm{CFU} / \mathrm{mL}$. The different columns correspond to different degrees of magnifications: $5 \times$ (left column), $40 \times$ (central column) by using a confocal microscope, and $>100 \times$ with SEM (right column).

and B. subtilis) and another group that does not induce any 193 responsive bending of the pillars in the center of the dried 194 patterns (S. epidermidis and L. sakei). These distinct behaviors 195 could be observed even by the naked eye in the form of a local 196 change in contrast at the surface (Figure 2, 5X). At higher 197 magnifications, the difference is clearly revealed to be 198 associated with the coalescence of adjacent pillars (Figure 2, 199 $40 \times$ and SEM $(100 \times))$.

We attempted to correlate these results to the general 201 characteristics of the bacterial species used in this work (Table $202 \mathrm{t} 1$ 1). Atomic force microscopy (AFM) imaging confirmed the $203 \mathrm{t} 1$ expected size and cell morphology for these bacteria: Gram- 204 negative (-) P. aeruginosa and E. coli as well as Gram-positive 205 (+) B. subtilis and L. sakei present a rod-like shape, while 206 Gram-positive (+) S. epidermidis has a spheroidal shape 207 (Figure S2). In addition, L. sakei and S. epidermidis are not 208 motile (no flagella present), while the other three strains have 209 flagella. From these considerations, we can conclude that the 210 different pattern types showed in Figure 2 (bending vs 211 nonbending) cannot be explained considering bacteria cell 212 
Table 1. General Characteristics of the Different Bacterial Strains Used in the Study ${ }^{a}$

\begin{tabular}{lclll}
\multicolumn{1}{c}{ strain } & gram & shape & \multicolumn{1}{c}{$L \times W_{\mathrm{a}}\left(\mu \mathrm{m}^{2}\right)$} & flagella \\
(a) P. aeruginosa & - & rod & $1.4( \pm 0.2) \times 0.8( \pm 0.2)$ & yes \\
(b) E. coli & - & $\operatorname{rod}$ & $1.7( \pm 0.2) \times 0.9( \pm 0.2)$ & yes \\
(c) B. subtilis & + & rod & $1.8( \pm 0.4) \times 0.80( \pm 0.2)$ & yes \\
(d) L. sakei & + & rod & $1.5( \pm 0.4) \times 0.8( \pm 0.2)$ & no \\
(e) S. epidermidis & + & spherical & $1.3( \pm 0.3) \times 1.3( \pm 0.3)$ & no
\end{tabular}

${ }^{a}$ AFM images of cells are presented in Figure S2.

213 morphology only. Similarly, the stiffness of the cell envelop 214 does not appear to play a critical role, with rigid Gram-positive 215 bacteria and softer Gram-negative bacteria distributed among 216 both pattern groups.

217 Interestingly, the different response of the microstructures 218 upon evaporation of the bacterial solutions correlates with the 219 presence or absence of flagella. Bacteria with flagella clearly 220 induce a bending response in the $\mathrm{H} 15$ pillars, while 221 nonflagellated bacteria are unable to bend the pillars when 222 used at the same bacterial concentration.

223 For the bacteria that induce a mechanical response, a 224 concentration dependence is observed, with deformation of 225 pillar clusters at the center of the dried droplet observed for 226 bacteria concentrations between $10^{7} \mathrm{CFU} / \mathrm{mL}$ and $10^{9} \mathrm{CFU} /$ $227 \mathrm{~mL}$, while none is observed for lower bacteria concentrations $228\left(10^{5} \mathrm{CFU} / \mathrm{mL}\right)$. At low concentrations, only the perimeter 229 near the corners of the dried square pattern presented 230 coalescence of the pillars (Figure $3 \mathrm{a}-\mathrm{c}$ ). This can be attributed 231 to the coffee-stain-like effect, able to drag bacterial cells toward 232 the droplet contact line, increasing the local concentration of 233 bacteria during evaporation. ${ }^{31}$ Interestingly, bacterial cells 234 without flagella confirm the absence of responsivity at different 235 cell concentrations (Figure $3 \mathrm{~d}-\mathrm{f}$ ).
No clear correlation was observed between bacterial species 236 and the cluster symmetries obtained (e.g., dimer, trimer, 237 tetramer, etc.). However, the data suggests that the assemblies 238 emerge due to perturbation of the balance between capillary 239 forces and elastic restoration forces in the presence of bacteria 240 with flagella. In the next section, we discuss a possible 241 mechanism for this distinctive behavior.

242

Possible Origin of Bacteria-Induced Coalescence. In 243 the previous sections, we determined the critical pillar aspect 244 ratio, below which surface tension forces were not able to 245 induce pillar coalescence in pure water. Interestingly, the 246 responsivity is dramatically enhanced when the droplets 247 contain flagellated bacteria. While the bending process at the 248 perimeter of the contact line appears similar in both cases, 249 coalescence within the central area is triggered at smaller 250 aspect ratios by the presence of bacteria with flagella. This 251 enhanced pillar bending effect results in characteristic patterns 252 on the substrate, distinct for motile and nonmotile bacteria. 253

The possible origin of the enhanced pillar bending may be 254 related to the ability of the bacteria with flagella to adhere to 255 more than one pillar (Figure S3), thus connecting adjacent 256 pillars and inducing a mechanical deformation. In the presence 257 of bacteria with flagella, we observed, at SEM, after drying, 258 structures bridging bent pillars, while nonflagellated bacteria 259 appeared attached to single pillars. The morphology of the 260 single bacterial cells cannot be distinguished, probably due to 261 distortions on the cell envelop after evaporation, in the absence 262 of fixation.

263

These effects can also be understood by comparing the 264 length scales of bacterial structures and pillar interspacing 265 distances. The average size of the capsule for a single bacterial 266 cell is below $2 \mu \mathrm{m}$ (Table 1), while flagella can reach tens of 267 $\mu \mathrm{m}$ beyond the outer cell membrane. ${ }^{37}$ Considering that in our 268 microstructured surfaces the interpillar distance was $5 \mu \mathrm{m}, 269$ bacteria without flagella will predominantly fall between the 270

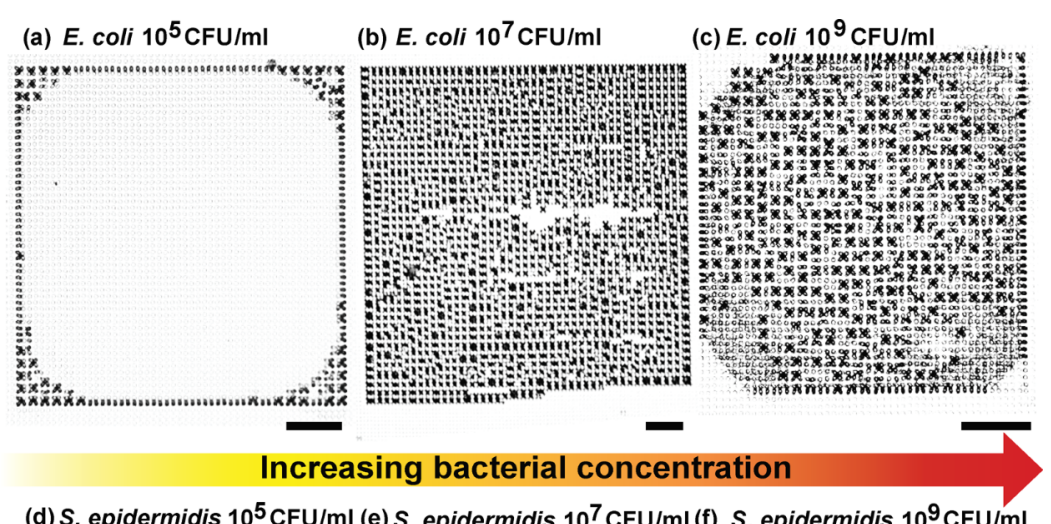

(d) S. epidermidis $10^{5} \mathrm{CFU} / \mathrm{ml}$ (e) $S$. epidermidis $10^{7} \mathrm{CFU} / \mathrm{ml}$ (f) $S$. epidermidis $10^{9} \mathrm{CFU} / \mathrm{ml}$

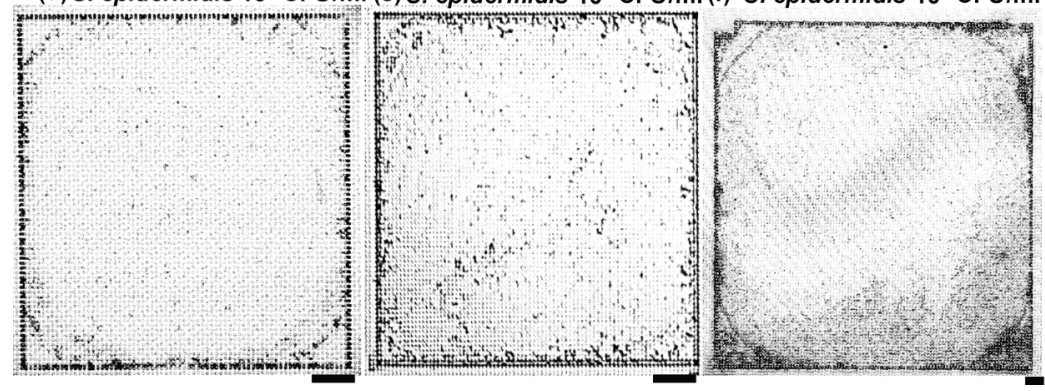

Figure 3. Effect of bacteria concentration on the bending pattern for E. coli and S. epidermidis on the H15 pillared substrate. Representative optical microscopy images for (a) $10^{5} \mathrm{CFU} / \mathrm{mL}$, (b) $10^{7} \mathrm{CFU} / \mathrm{mL}$, and (c) $10^{9} \mathrm{CFU} / \mathrm{mL}$. Scale bar in panels a-f is $100 \mu \mathrm{m}$. 
271 pillars or strongly adhere ${ }^{38}$ to single pillars. On the other hand, 272 bacteria with flagella, ${ }^{32}$ in which appendage sizes exceed the 273 interpillar distance, can potentially interact with more than one 274 pillar, leading to the observed pillar deformation.

275 In support of this, we found evidence of bacterial matter 276 residing between the bent pillars, after complete evaporation of 277 droplets containing flagellated bacteria (Figure 4). Non278 flagellated bacteria, on the other hand, are found attached to 279 individual pillars only, forming nonconnecting structures (see 280 Figures S4-S7).

\section{B. subtilis}

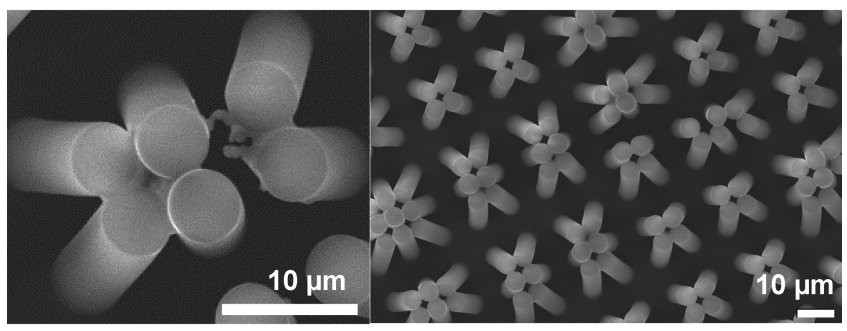

E. coli

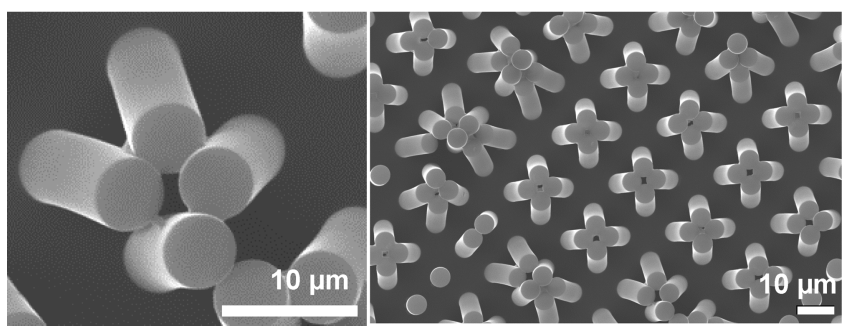

Figure 4. Representative SEM images of $\mathrm{H} 15$ pillared structures after drying of bacterial suspensions, showing motile bacteria (B. subtilis and E. coli) bridging the bent pillars. The concentration of the different bacterial species is $10^{7} \mathrm{CFU} / \mathrm{mL}$.

281 Although a more detailed investigation of bacterial behavior 282 during the actual drying process is necessary to confirm the 283 hypothesis proposed, our results support the potential use of 284 pillared soft substrates to discriminate between motile and 285 nonflagellated bacteria using a cost-effective and immediate 286 assay based on droplet-drying, which can be performed and 287 quickly analyzed by the naked eye. In addition, discrimination 288 of bacterial concentration is also possible, with only samples 289 containing concentrations above a critical threshold producing 290 a response. We envision that by tuning the properties of the 291 substrates, a more subtle differentiation between different 292 microorganisms and different bacterial concentrations could be 293 achieved in the future with this presented novel, easy to 294 fabricate, and cost-effective technology.

\section{CONCLUSIONS}

296 We show that soft micropillared surfaces can be tailor-made 297 sensitive to the presence of isolated bacterial cells in a single 298 drop. The evaporation of water droplets and bacterial 299 suspensions over fabricated micropillar arrays leads to very 300 distinct micropillar deformations and patterns. Once the 301 threshold for elastocapillary pillar coalescence is found, we 302 observe that only bacteria with flagella can promote pillar 303 coalescence. Such responsive micropillared surfaces could 304 provide a platform for the development of fast and cost305 effective self-responsive surfaces for bacterial detection and 306 differentiation.

\section{EXPERIMENTS AND METHODS}

The epoxy micropillars were fabricated by casting EPO-TEK OG142- 308 13 from Epoxy Technology into a negative replica PDMS mold, as 309 described. ${ }^{31,32}$ After the resin was casted, a $1.1 \mathrm{~mm}$ thick glass slide 310 was placed over the mold and placed below an ultraviolet light for 20311 min until the epoxy pillar was cured. The epoxy micropillars were 312 mechanically removed from the mold. The SEM images of the epoxy 313 pillars are shown in Figure S1. After the sample preparation, we 314 measured the Young modulus $(E)$ of the bulk material and the 315 micropillar via an axial compression test. The $E$ value for the bulk 316 material was $1 \pm 0.3 \mathrm{GPa}$, and the $E$ value for the $\mathrm{H} 15$ substrate was 317 $0.5 \pm 0.2 \mathrm{GPa}$.

Bacterial cultures were performed following recommended growing 319 conditions for each species. P. aeruginosa ATCC-8626, E. coli ATCC- 320 10798, and S. epidermidis ATTC-12228 were grown overnight at 37321 ${ }^{\circ} \mathrm{C}$ in liquid broth medium (Oxoid Ltd., Thermo Fisher). B. subtilis 322 subsp. subtilis ATCC-6051 and L. sakei DSMZ-20017 were grown 323 overnight at $30^{\circ} \mathrm{C}$ in MRS broth medium from Oxoid Ltd., Thermo 324 Fisher. All of the cells cultures were then centrifuged and redispersed 325 in sterile deionized water two times, finally adjusting the bacterial 326 concentration to $10^{7}$ colony-forming units per milliliter $(\mathrm{CFU} / \mathrm{mL}), 327$ unless differently specified. Note that colony counting was performed 328 after cell redispersion in deionized water to ensure cell viability. 329

The evaporation of all droplets was carried out placing a droplet of 330 $5-10 \mu \mathrm{L} \pm 4 \mu \mathrm{L}$ on the epoxy substrates. For droplets containing 331 bacteria, experiments were performed in triplicates drying 5 droplets 332 over substrates independently. The images were collected with a 333 CMOS camera PCO Sensicam at 1 frames per second (fps). The 334 droplet completely evaporated in approximately $2100 \pm 300$ s. 335 Evaporation experiments were assessed at room temperature $(21 \pm 3336$ ${ }^{\circ} \mathrm{C}$ ) in an atmosphere with a relative humidity of $35 \pm 5 \%$. 337

The contact angle measurements of water and bacterial suspension 338 droplets on epoxy surfaces were carried out by placing a water droplet 339 with bacterial suspension of $10^{7} \mathrm{CFU} / \mathrm{mL}$ on the epoxy substrates. 340 The contact angle (CA) for $\mathrm{H} 15$ was $100^{\circ} \pm 7^{\circ}$, whereas the CA was 341 $92^{\circ} \pm 5^{\circ}$ for $\mathrm{H} 22, \mathrm{H} 28$, and $\mathrm{H} 33$. For longer pillars like $\mathrm{H} 38$ and 342 $\mathrm{H} 45$, the CA was $88^{\circ} \pm 3^{\circ}$. CA hysteresis was carried out in a similar 343 manner as CA measurements but by tilting the substrate $45^{\circ} .344$ Experiments were performed for the H15 substrate with and without 345 bacterial containing droplets only, the CA hysteresis was $50^{\circ} \pm 8^{\circ}$. No 346 significant differences in CA and CA hysteresis were observed 347 between water droplets and the deposited bacterial containing 348 droplets. CA values are shown in Table S1.

Transmission light microscopy images of the dried patterns were 350 collected with a Zeiss 510 confocal microscope equipped with $\times 10,351$ $\times 20$, and $\times 40$ air objectives. AFM measurements from the Supporting 352 Information were obtained using a Bruker Multimode 8 and a 353 Keysights 5500 instrument. Prior to AFM morphological analysis, a 354 droplet of bacteria suspension $\left(10^{7} \mathrm{CFU} / \mathrm{mL}\right)$ was deposited onto an 355 oxygen plasma-treated epoxy flat substrate and dried at room 356 temperature. Estimated length $(L) \times$ width $\left(W_{\mathrm{a}}\right)$ in Table 1 are 357 reported within a standard deviation of $10-25 \%$ obtained by 358 measuring 15-20 cells per bacterial strains. These tests were carried 359 out independently in triplicates. Top-view scanning electron 360 microscopy (SEM) imaging was performed at $20 \mathrm{kV}$. Side-view 361 SEM was recorded after fracturing the epoxy/glass with a diamond 362 cutter at accelerating voltages of $3 \mathrm{kV}$. Prior to SEM inspection in a 363 JSM-6610 JEOL system, all samples were coated with $20 \mathrm{~nm}$ of 364 chromium to increase the electrical conductivity. SEM images are 365 presented without fixation, which involves several solvent exchange 366 steps ${ }^{39}$ preserving the bacterial footprints after droplet evaporation. 367

\section{ASSOCIATED CONTENT}

\section{S Supporting Information}

The Supporting Information is available free of charge on the 370 ACS Publications website at DOI: 10.1021/acsabm.8b00176. 371

SEM images of some of the pillared arrays fabricated; 372 AFM images of bacterial cells dried over flat epoxy 373 
382 AUTHOR INFORMATION

\section{Corresponding Authors}

384 *E-mail: A.Susarrey-Arce@liverpool.ac.uk.

385 *E-mail: Jose.HernandezSanchez@kaust.edu.sa.

386 *E-mail: j.h.snoeijer@utwente.nl.

387 *E-mail: r.raval@liverpool.ac.uk.

\section{ORCID}

389 Arturo Susarrey-Arce: 0000-0003-2572-223X

390 Detlef Lohse: 0000-0003-4138-2255

391 Han Gardeniers: 0000-0003-0581-2668

\section{Author Contributions}

${ }_{393}$ OA.S.-A. and J.F.H.-S. contributed equally to this work

\section{Notes}

395 The authors declare no competing financial interest.

\section{ACKNOWLEDGMENTS}

397 We would like to thank Dr. Joanna Wnetrzak and the 398 Liverpool Centre for Cell Imaging (CCI) for help with 399 experimental design and technical support. We also acknowl400 edge the support of the Nanoinvestigation Centre at University 401 of Liverpool (NICAL) for access to the SEM facility. Stefan 402 Schlautmann (Mesoscale Chemical Systems, MESA+ Institute 403 of Nanotechnology, University of Twente) is also acknowl404 edged for sample fabrication. This work was partly funded by 405 BBSRC (BB/R012415/1).

\section{REFERENCES}

407 (1) Tawfick, S.; De Volder, M.; Copic, D.; Park, S. J.; Oliver, C. R.; 408 Polsen, E. S.; Roberts, M. J.; Hart, A. J. Engineering of Micro-and 409 Nanostructured Surfaces with Anisotropic Geometries and Properties. 410 Adv. Mater. 2012, 24, 1628-1674.

411 (2) Le, V.; Lee, J.; Chaterji, S.; Spencer, A.; Liu, Y.-L.; Kim, P.; Yeh, 412 H.-C.; Kim, D.-H.; Baker, A. B. Syndecan-1 in Mechanosensing of 413 Nanotopological Cues in Engineered Materials. Biomaterials 2018, 414 155, 13-24.

415 (3) Chakrapani, N.; Wei, B.; Carrillo, A.; Ajayan, P. M.; Kane, R. S. 416 Capillarity-Driven Assembly of Two-Dimensional Cellular Carbon 417 Nanotube Foams. Proc. Natl. Acad. Sci. U. S. A. 2004, 101, 40094184012 .

419 (4) Boesel, L. F.; Greiner, C.; Arzt, E.; Del Campo, A. Gecko420 Inspired Surfaces: a Path to Strong and Reversible Dry Adhesives. 421 Adv. Mater. 2010, 22, 2125-2137.

422 (5) Prieto-López, L.; Williams, J. Using Microfluidics to Control Soft 423 Adhesion. J. Adhes. Sci. Technol. 2016, 30, 1555-1573.

424 (6) Prieto-López, L. O.; Williams, J. A. Switchable Adhesion Surfaces 425 with Enhanced Performance Against Rough Counterfaces. Biomimetics 426 2016, 1, 2.

427 (7) Kwak, M. K.; Jeong, H.-E.; Kim, T.-i.; Yoon, H.; Suh, K. Y. Bio428 Inspired Slanted Polymer Nanohairs for Anisotropic Wetting and 429 Directional Dry Adhesion. Soft Matter 2010, 6, 1849-1857.

430 (8) Trichet, L.; Le Digabel, J.; Hawkins, R. J.; Vedula, S. R. K.; 431 Gupta, M.; Ribrault, C.; Hersen, P.; Voituriez, R.; Ladoux, B. 432 Evidence of a Large-Scale Mechanosensing Mechanism for Cellular 433 Adaptation to Substrate Stiffness. Proc. Natl. Acad. Sci. U. S. A. 2012, 434 109, 6933-6938.
(9) Liu, K.; Jiang, L. Bio-Inspired Design of Multiscale Structures for 435 Function Integration. Nano Today 2011, 6, 155-175. (10) Asayesh, F.; Zarabadi, M. P.; Greener, J. A New Look at 437 Bubbles During Biofilm Inoculation Reveals Pronounced Effects on 438 Growth and Patterning. Biomicrofluidics 2017, 11, 064109.

(11) Dean, B.; Bhushan, B. Shark-Skin surfaces for Fluid-Drag 440 Reduction in Turbulent Flow: a Review. Philos. Trans. R. Soc., A 2010, 441 368, 4775-4806.

(12) Guo, Z.; Liu, W. Biomimic from the Superhydrophobic Plant 443 Leave in Nature: Binary Structure and Unitary Structure. Plant Sci. 444 2007, 172, 1103-1112.

445

(13) Feng, L.; Zhang, Y.; Cao, Y.; Ye, X.; Jiang, L. The Effect of 446 Surface Microstructures and Surface Compositions on the Wett- 447 abilities of Flower Petals. Soft Matter 2011, 7, 2977-2980. 448

(14) Fratzl, P.; Barth, F. G. Biomaterial Systems for Mechanosensing 449 and Actuation. Nature 2009, 462, 442-448.

450

(15) Bico, J.; Roman, B.; Moulin, L.; Boudaoud, A. Adhesion: 451 Elastocapillary Coalescence in Wet Hair. Nature 2004, 432, 690-690. 452

(16) Yeh, Y.-H.; Cho, K.-H.; Chen, L.-J. Effect of Softness of 453 Polydimethylsiloxane on the Hydrophobicity of Pillar-Like Patterned 454 Surfaces. Soft Matter 2012, 8, 1079-1086.

(17) Nill, P.; Goehring, N; Loeffler, R; Peschel, A; Kern, D. P. 456 Studying Bacterial Adhesion Forces: Staphylococcus Aureus on 457 Elastic Poly (Dimethyl) Siloxane Substrates. Microelectron. Eng. 458 2011, 88, 1825-1827.

(18) Pokroy, B.; Kang, S. H.; Mahadevan, L.; Aizenberg, J. Self- 460 Organization of a Mesoscale Bristle Into Ordered, Hierarchical 461 Helical Assemblies. Science 2009, 323, 237-240. 462

(19) Chandra, D.; Yang, S. Stability of High-Aspect-Ratio Micro- 463 pillar Arrays Against Adhesive and Capillary Forces. Acc. Chem. Res. 464 2010, 43, 1080-1091.

(20) Chandra, D.; Yang, S. Capillary-Force-Induced Clustering of 466 Micropillar Arrays: is it Caused by Isolated Capillary Bridges or by the 467 Lateral Capillary Meniscus Interaction Force? Langmuir 2009, 25, 468 10430-10434.

(21) Roman, B.; Bico, J. Elasto-Capillarity: Deforming an Elastic 470 Structure with a Liquid Droplet. J. Phys.: Condens. Matter 2010, 22, 471 493101.

(22) Marchand, A.; Weijs, J. H.; Snoeijer, J. H.; Andreotti, B. Why is 473 Surface Tension a Force Parallel to the Interface? Am. J. Phys. 2011, 474 79, 999-1008.

475

(23) Weijs, J. H.; Andreotti, B.; Snoeijer, J. H. Elasto-Capillarity at 476 the Nanoscale: on the Coupling between Elasticity and Surface 477 Energy in Soft Solids. Soft Matter 2013, 9, 8494-8503.

(24) Yang, M. T.; Fu, J.; Wang, Y.-K.; Desai, R. A.; Chen, C. S. 479 Assaying Stem Cell Mechanobiology on Microfabricated Elastomeric 480 Substrates with Geometrically Modulated Rigidity. Nat. Protoc. 2011, 481 6, 187-213.

(25) Wei, Z.; Schneider, T.; Kim, J.; Kim, H.-Y.; Aizenberg, J.; 483 Mahadevan, L. Elastocapillary Coalescence of Plates and Pillars. Proc. 484 R. Soc. London, Ser. A 2015, 471, 20140593.

(26) Ledesma-Aguilar, R.; Laghezza, G.; Yeomans, J. M.; Vella, D. 486 Using Evaporation to Control Capillary Instabilities in Micro- 487 Systems. Soft Matter 2017, 13, 8947-8956.

(27) Jahed, Z.; Shahsavan, H.; Verma, M. S.; Rogowski, J. L.; Seo, B. 489 B.; Zhao, B.; Tsui, T. Y.; Gu, F. X.; Mofrad, M. R. K. Bacterial 490 Networks on Hydrophobic Micropillars. ACS Nano 2017, 11, 675- 491 683.

(28) Chew, S. C.; Kundukad, B.; Teh, W. K.; Doyle, P.; Yang, L.; 493 Rice, S. A.; Kjelleberg, S. Mechanical Signatures of Microbial Biofilms 494 in Micropillar-Embedded Growth Chambers. Soft Matter 2016, 12, 495 $5224-5232$.

(29) Biais, N.; Ladoux, B.; Higashi, D.; So, M.; Sheetz, M. 497 Cooperative Retraction of Bundled Type IV Pili Enables Nanonewton 498 Force Generation. PLoS Biol. 2008, 6, 1-7.

(30) Ng, D.; Harn, T.; Altindal, T.; Kolappan, S.; Marles, J. M.; Lala, 500 R.; Spielman, I.; Gao, Y.; Hauke, C. A.; Kovacikova, G.; Verjee, Z.; 501 Taylor, R. K.; Biais, N.; Craig, L. The Vibrio cholerae Minor Pilin 502 
503 TcpB Initiates Assembly and Retraction of the Toxin-Coregulated 504 Pilus. PLoS Pathog. 2016, 12, 1-31.

505 (31) Susarrey-Arce, A.; Marin, A.; Massey, A.; Oknianska, A.; Díaz506 Fernandez, Y.; Hernández-Sánchez, J. F.; Griffiths, E.; Gardeniers, J. 507 G. E.; Snoeijer, J. H.; Lohse, D.; Raval, R. Pattern Formation by 508 Staphylococcus Epidermidis via Droplet Evaporation on Microoillars 509 Arrays at a Surface. Langmuir 2016, 32, 7159-7169.

510 (32) Hochbaum, A. I.; Aizenberg, J. Bacteria Pattern Spontaneously 511 on Periodic Nanostructure Arrays. Nano Lett. 2010, 10, 3717-3721. 512 (33) Li, X.; Cheung, G. S.; Watson, G. S.; Watson, J. A.; Lin, S.; 513 Schwarzkopf, L.; Green, D. W. The Nanotipped Hairs of Gecko Skin 514 and Biotemplated Replicas Impair and/or Kill Pathogenic Bacteria 515 with High Efficiency. Nanoscale 2016, 8, 18860-18869.

516 (34) Kim, P.; Epstein, A. K.; Khan, M.; Zarzar, L. D.; Lipomi, D. J.; 517 Whitesides, G. M.; Aizenberg, J. Structural Transformation by 518 Electrodeposition on Patterned Substrates (STEPS): A New Versatile 519 Nanofabrication Method. Nano Lett. 2012, 12, 527-533.

520 (35) Pokroy, B.; Epstein, A. K.; Persson-Gulda, M. C. M.; Aizenberg, $521 \mathrm{~J}$. Fabrication of Bioinspired Actuated Nanostructures with Arbitrary 522 Geometry and Stiffness. Adv. Mater. 2009, 21, 463-469.

523 (36) Hadjittofis, A.; Lister, J. R.; Singh, K.; Vella, D. Evaporation 524 Effects in Elastocapillary Aggregation. J. Fluid Mech. 2016, 792, 168525185.

526 (37) Haiko, J.; Westerlund-Wikström, B. The Role of the Bacterial 527 Flagellum in Adhesion and Virulence. Biology 2013, 2, 1242-1267.

528 (38) Hizal, F.; Choi, C.-H.; Busscher, H. J.; van der Mei, H. C. 529 Staphylococcal Adhesion, Detachment and Transmission on Nano530 pillared Si Surfaces. ACS Appl. Mater. Interfaces 2016, 8, 3043053130439 .

532 (39) Susarrey-Arce, A.; Sorzabal-Bellido, I.; Oknianska, A.; McBride, 533 F.; Beckett, A. J.; Gardeniers, J. G. E.; Raval, R.; Tiggelaar, R. M.; Diaz 534 Fernandez, Y. A. Bacterial Viability on Chemically Modified Silicon 535 Nanowire Arrays. J. Mater. Chem. B 2016, 4, 3104-3112. 